\title{
A simple status quo that ensures participation (with application to efficient bargaining)
}

\author{
ILYA SEGAL \\ Department of Economics, Stanford University \\ Michael D. Whinston \\ Department of Economics, Northwestern University
}

\begin{abstract}
We consider Bayesian incentive-compatible mechanisms with independent types and either private values or interdependent values that satisfy a form of "congruence." We show that in these settings, interim participation constraints are satisfied when the status quo is the randomized allocation that has the same distribution as the equilibrium allocation in the mechanism. Moreover, when utilities are convex in the allocation, we can instead satisfy participation constraints with the deterministic status quo equal to the expected equilibrium allocation in the mechanism. For quasilinear settings, these observations imply the possibility of efficient bargaining when the status quo specifies the expected efficient decision provided that the total surplus is convex in the decision.

KEYWORDs. Efficient property rights, asymmetric information bargaining, transaction costs.

JEL Classification. C78, D23, D44.
\end{abstract}

\section{InTRODUCTION}

Myerson and Satterthwaite (1983) first demonstrated, in a bilateral trading setting, that participation constraints may make it impossible for parties who have private information to bargain to fully efficient trade. Specifically, they showed that when a seller owns a good that a buyer may wish to purchase, and the parties' privately known valuations are statistically independent, under very weak conditions no efficient, Bayesian incentivecompatible and budget-balanced mechanism can satisfy the parties' interim participation constraints. ${ }^{1}$

Ilya Segal: ilya.segal@stanford.edu

Michael D. Whinston: mwhinston@northwestern.edu

This paper supercedes the authors' earlier working paper "An Expected-Efficient Status Quo Allows Efficient Bargaining." The authors thank Bengt Holmstrom, Ron Siegel, three anonymous referees, Martin Osborne (the editor), and seminar participants at ASU, Cambridge, IDEI, LSE, Northwestern, Oxford, UCL, and the BU conference on "New Directions in Organization Theory" for helpful comments. Segal thanks the Toulouse Network for Information Technology for financial support. Whinston thanks the Toulouse Network for Information Technology and the Leverhulme Trust for financial support, and Nuffield College and Oxford University's Department of Economics for their hospitality.

${ }^{1}$ Similar results in the context of public goods are established by Laffont and Maskin (1979) and Mailath and Postlewaite (1990) (the former under a restriction to differentiable mechanisms).

Copyright $\odot 2011$ Ilya Segal and Michael D. Whinston. Licensed under the Creative Commons AttributionNonCommercial License 3.0. Available at http: //econtheory . org.

DOI: 10.3982/TE591 
Subsequently, the literature has asked whether efficient bargaining can arise for some status quo allocations (that is, allocations of ex ante "property rights"). Cramton et al. (1987) first showed that in the problem of allocating a divisible good to the highest value agent, efficiency can be achieved with symmetric agents when the status quo allocation of the good is close enough to equal shares. Schmitz (2002), Che (2006), Figueroa and Skreta (2007), Yenmez (2007), and Gershkov and Schweinzer (2010) provide other examples in which efficient bargaining is possible. Schweizer (2006, Proposition 2) establishes the existence of an efficiency-permitting status quo allocation in a more general model than the ones studied in the papers cited above. Neeman (1999), Ornelas and Turner (2007), and Turner (2008) construct examples in which an efficiency-permitting status quo allocation does not exist. Fieseler et al. (2003) and Jehiel and Pauzner (2006) study efficiency-permitting status quo allocations in some settings with interdependent values.

This paper describes a simple status quo allocation that assures the satisfaction of participation constraints in a very general model. Namely, we study Bayesian incentivecompatible mechanisms with independent types and either private values or interdependent values that satisfy a form of "congruence." We show that in any such mechanism, interim participation constraints are satisfied when the status quo is the randomized allocation that has the same distribution as the equilibrium allocation in the mechanism. Furthermore, when utilities are convex in the allocation, we can instead satisfy participation constraints with the deterministic status quo equal to the expected equilibrium allocation in the mechanism.

For the case of quasilinear utilities, these observations imply the possibility of efficient bargaining when the status quo specifies the expected efficient decision, in a setting that strictly generalizes all the preceding settings for which the existence of an efficiency-permitting status quo has been shown. In contrast to Schweizer (2006), it also describes a natural status quo that permits efficient bargaining: the expected efficient decision. Moreover, the proof of the result is surprisingly simple.

Our general results apply more broadly, however, both to nonquasilinear environments and to implementation of ex post inefficient outcomes. For example, precommitment to ex post inefficient outcomes may be desirable in strategic settings, when incentives for ex ante investments need to be created, or when distributional or risksharing concerns are present.

\section{THE GENERAL MODEL AND RESUlT}

Let $Y$ be the set of feasible allocations, assumed to be a measurable space (an allocation may include monetary transfers). Let $I$ be a finite set of agents. Each agent $i \in I$ privately observes his type $\theta_{i}$, which is a realization of a random variable $\widetilde{\theta}_{i}$ in a measurable space $\Theta_{i}$. The agents' types are stochastically independent of each other. We let $\Theta=\Theta_{1} \times \cdots \times \Theta_{I}$. Each agent $i \in I$ is an expected utility maximizer, with a measurable Bernoulli utility function $u_{i}: Y \times \Theta \rightarrow \mathbb{R}$. We consider the implementation of an allocation rule $\eta: \Theta \rightarrow Y$, which we restrict to be measurable and satisfy 
$\sup _{i \in I, \theta, \theta^{\prime} \in \Theta}\left|u_{i}\left(\eta\left(\theta^{\prime}\right), \theta\right)\right|<\infty .^{2}$ By the Revelation Principle, we focus on the direct revelation mechanism implementing allocation rule $\eta$.

Much of the literature has focused on settings with private values, in which $u_{i}(y, \theta)$ depends on $\theta$ only through agent $i$ 's type $\theta_{i}$. Our results will apply not only to this case, but also to more general settings with interdependent values in which allocation rules satisfy the following property:

Definition 1. Allocation rule $\eta$ is cross-congruent (CC) if for all $i \in I$ and all $\theta_{i}^{\prime}, \theta_{i} \in \Theta_{i}$, for any random variable $\widetilde{\widetilde{\theta}}_{-i}$ that has the same distribution as $\widetilde{\theta}_{-i}$ and is independent of it, we have $\mathbb{E}\left[u_{i}\left(\eta\left(\theta_{i}^{\prime}, \widetilde{\theta}_{-i}\right), \theta_{i}, \widetilde{\theta}_{-i}\right)\right] \geq \mathbb{E}\left[u_{i}\left(\eta\left(\theta_{i}^{\prime}, \widetilde{\widetilde{\theta}}_{-i}\right), \theta_{i}, \widetilde{\theta}_{-i}\right)\right]$.

In words, CC states that each agent $i$ 's interim expected utility cannot be raised (regardless of whether he reports truthfully) when each of the other agents switches from truthful reporting to randomizing over his report independently of his true type but with the correct distribution. ${ }^{3}$ With private values, any allocation rule is CC, since an agent cares only about the other agents' reports and not their true types, and so the inequality in the definition holds with equality. Some interdependent-value settings in which CC holds will be described in Section 3.2 below.

The Bayesian incentive-compatibility (IC) constraints in the direct revelation mechanism $\eta$ take the form

$$
\mathbb{E}\left[u_{i}\left(\eta\left(\theta_{i}, \widetilde{\theta}_{-i}\right), \theta_{i}, \widetilde{\theta}_{-i}\right)\right] \geq \mathbb{E}\left[u_{i}\left(\eta\left(\theta_{i}^{\prime}, \widetilde{\theta}_{-i}\right), \theta_{i}, \widetilde{\theta}_{-i}\right)\right] \quad \text { for all } i \in I, \theta_{i}, \theta_{i}^{\prime} \in \Theta_{i}
$$

In general, we allow status quo allocations $\tilde{y}$ that are random variables on $Y$ and independent of the true state $\widetilde{\theta}$. The agents' (interim) individual rationality, or participation, constraints (IR) then take the form ${ }^{4}$

$$
\mathbb{E}\left[u_{i}\left(\eta\left(\theta_{i}, \widetilde{\theta}_{-i}\right), \theta_{i}, \widetilde{\theta}_{-i}\right)\right] \geq \mathbb{E}\left[u_{i}\left(\tilde{y}, \theta_{i}, \widetilde{\theta}_{-i}\right)\right] \quad \text { for all } i \in I, \theta_{i} \in \Theta_{i}
$$

In some cases, instead of a randomized status quo, we can use a deterministic one, which implements a given allocation from $Y$ with probability 1.

Proposition 1. Suppose that the mechanism $\eta$ satisfies IC and CC.

(a) Then mechanism $\eta$ also satisfies IR when the status quo is the randomized allocation $\tilde{y}$ that has the same distribution as the equilibrium allocation $\eta(\widetilde{\theta})$ in the mechanism.

\footnotetext{
${ }^{2}$ These assumptions ensure that all the expectations below exist and Fubini's Theorem (i.e., the law of iterated expectations) applies. The boundedness assumption could be relaxed substantially.

${ }^{3}$ In particular, for a Bayesian incentive-compatible mechanism $\eta$, this condition implies that each agent prefers all the agents to report their types truthfully rather than to randomize over their reports with the correct distributions but independently of the true types, which motivates the name "cross-congruence."

${ }^{4}$ We follow the literature described in the Introduction by focusing on mechanisms in which any agent's refusal to participate enforces the status quo. Note that if the mechanism could instead impose externalities on nonparticipating agents (as in Segal 1999), this could relax the participation constraints.
} 
(b) If, in addition, the allocation space $Y$ is a convex set in a topological vector space and the Bernoulli utility function $u_{i}(y, \theta)$ of each agent $i \in I$ is convex in the allocation $y \in Y$ for each state $\theta \in \Theta$, then mechanism $\eta$ also satisfies IR with the deterministic status quo equal to the expected equilibrium allocation in the mechanism, $\mathbb{E}[\eta(\widetilde{\theta})]$.

Proof. For part (a), for all $i \in I$ and $\theta_{i} \in \Theta_{i}$, letting $\widetilde{\widetilde{\theta}}_{=}\left(\widetilde{\widetilde{\theta}}_{i}, \widetilde{\widetilde{\theta}}_{-i}\right)$ be a random variable equal in distribution to $\widetilde{\theta}$ and independent of it, we can write

$$
\begin{aligned}
\mathbb{E}\left[u_{i}\left(\eta\left(\theta_{i}, \widetilde{\theta}_{-i}\right), \theta_{i}, \widetilde{\theta}_{-i}\right)\right] & \geq \mathbb{E}\left[u_{i}\left(\eta\left(\widetilde{\widetilde{\theta}}_{i}, \widetilde{\theta}_{-i}\right), \theta_{i}, \widetilde{\theta}_{-i}\right)\right] \\
& \geq \mathbb{E}\left[u_{i}\left(\eta\left(\widetilde{\widetilde{\theta}}_{i}, \widetilde{\widetilde{\theta}}_{-i}\right), \theta_{i}, \widetilde{\theta}_{-i}\right)\right]=\mathbb{E}\left[u_{i}\left(\widetilde{y}, \theta_{i}, \widetilde{\theta}_{-i}\right)\right] .
\end{aligned}
$$

The first inequality follows from IC, while the second inequality follows from CC.

For part (b), observe in addition that by Jensen's inequality and iterated expectations, $\mathbb{E}\left[u_{i}\left(\eta(\widetilde{\widetilde{\theta}}), \theta_{i}, \widetilde{\theta}_{-i}\right)\right] \geq \mathbb{E}\left[u_{i}\left(\mathbb{E}[\eta(\widetilde{\widetilde{\theta}})], \theta_{i}, \widetilde{\theta}_{-i}\right)\right]$.

In words, IC implies, in particular, that an agent of any given type could not gain by misreporting his type randomly in a way that mimics his type distribution. Under CC, his expected payoff could only be lowered further by replacing the other agents' reports with random draws from their distributions that are independent of their true types. Thus, we obtain a randomized status quo that yields the same distribution over allocations as the equilibrium distribution of the mechanism and satisfies all of the participation constraints, proving Proposition 1(a). Proposition 1(b) allows us to replace the randomized status quo with its expectation when the agents are weakly risk-loving with respect to the allocation.

Note that the status quo constructed in Proposition 1(a) satisfies any constraints that are satisfied by the mechanism itself. The same holds for the deterministic status quo constructed in Proposition 1(b) for constraints that are convex and state-independent, such as budget balance, resource nonwastefulness, or bounds on consumption or transfers.

\section{APPLICATION TO BARGAINING IN QUASILINEAR ENVIRONMENTS}

We now apply Proposition 1 to bargaining in quasilinear environments and, in particular, to the possibility of efficient bargaining, as examined in the literature discussed in the Introduction. For this purpose, we let the allocation space be $Y=X \times \mathbb{R}^{I}$, so that an allocation $y=(x, t)$ consists of a nonmonetary decision $x \in X$ and a profile $t \in \mathbb{R}^{I}$ of monetary transfers to the agents. We say that a transfer vector $t \in \mathbb{R}^{I}$ is budget-balanced if $\sum_{i \in I} t_{i}=0$. The utility of each agent $i$ takes the quasilinear form $u_{i}((x, t), \theta)=v_{i}(x, \theta)+t_{i}$. We assume that $X$ is a measurable space and that the functions $v_{i}$ are measurable and uniformly bounded (i.e., $\sup _{i \in I, x \in X, \theta \in \Theta}\left|v_{i}(x, \theta)\right|<\infty$ ). The total surplus from decision $x$ in state $\theta$ is $s(x, \theta) \equiv \sum_{i \in I} v_{i}(x, \theta)$. A direct revelation mechanism in this setting can be written as $\eta=\langle\chi, \tau\rangle$, where $\chi: \Theta \rightarrow X$ is the decision rule and $\tau: \Theta \rightarrow \mathbb{R}^{I}$ is the transfer rule. We restrict both functions to be measurable and 
the transfers $\tau$ to be bounded. ${ }^{5}$ Note that the satisfaction of property CC in the mechanism $\langle\chi, \tau\rangle$ is entirely determined by its decision rule $\chi$.

The literature on efficient bargaining is interested in efficient decision rules, that is, rules for which

$$
\chi^{*}(\theta) \in \underset{x \in X}{\arg \max } s(x, \theta) \quad \text { for all } \theta \in \Theta .
$$

In line with the results of Section 2, we begin with the more general question concerning the possibility of implementing an arbitrary given decision rule $\chi$ in a mechanism that is IC, IR, and, in addition, satisfies the budget-balance (BB) condition

$$
\sum_{i \in I} \tau_{i}(\theta)=0 \text { for all } \theta \in \Theta
$$

Following the insight of Arrow (1979) and d'Aspremont and Gérard-Varet (1979), starting with any IC mechanism $\langle\chi, \tau\rangle$, we can construct another IC mechanism $\langle\chi, \bar{\tau}\rangle$ that also satisfies (BB), by taking the new transfers to be

$$
\bar{\tau}_{i}(\theta)=\mathbb{E}\left[\tau_{i}\left(\theta_{i}, \widetilde{\theta}_{-i}\right)\right]-\frac{1}{|I|-1} \sum_{j \in I \backslash\{i\}} \mathbb{E}\left[\tau_{j}\left(\theta_{j}, \widetilde{\theta}_{-j}\right)\right] .
$$

Furthermore, if the decision rule $\chi$ satisfies CC, then Proposition 1(a) yields a randomized status quo for which mechanism $(\chi, \bar{\tau})$ also satisfies IR. Finally, under convexity of agents' utilities in the decision and of the decision space $X$, Proposition $1(\mathrm{~b})$ implies that IR can be satisfied with a deterministic status quo $(\hat{x}, \hat{t})$ specifying decision $\hat{x}=\mathbb{E}[\chi(\widetilde{\theta})]$. Note that since the mechanism $(\chi, \bar{\tau})$ satisfies BB, the status quo transfers $\hat{t}$ obtained in this way are budget-balanced as well.

Now we establish a somewhat stronger result: the deterministic status quo $\hat{x}=$ $\mathbb{E}[\chi(\widetilde{\theta})]$ can be used under the weaker assumption that only the total surplus $s(x, \theta)$ is convex in the decision. We do so in a manner designed to facilitate comparison to the existing literature, which is discussed further in Section 4. We begin with the following definition.

Definition 2. Status quo decision $\hat{x} \in X$ sustains decision rule $\chi$ in bargaining if there exists an IC BB mechanism $\langle\chi, \tau\rangle$ that satisfies IR for some status quo allocation $(\hat{x}, \hat{t})$ with budget-balanced transfers $\hat{t} \in \mathbb{R}^{I}$. Status quo decision $\hat{x} \in X$ permits efficient bargaining if it sustains an efficient decision rule $\chi^{*}$ in bargaining.

Note that it does not matter which budget-balanced status quo transfers $\hat{t}$ are used in the definition, since the mechanism's transfer rule $\tau$ can always be adjusted to $\hat{\tau}_{i}(\theta)=$ $\tau_{i}(\theta)-\hat{t}_{i}$ to preserve BB and IC and satisfy IR for the status quo $(\hat{x}, 0)$. Hence for definiteness, we focus on status quos with no transfers.

\footnotetext{
${ }^{5}$ These restrictions ensure the existence of the expectations and the infimum below.
} 
Given an IC mechanism $\langle\chi, \tau\rangle$, for any $\hat{x} \in X$ and $\hat{\theta}=\left(\hat{\theta}_{1}, \ldots, \hat{\theta}_{I}\right) \in \Theta$, define

$$
\begin{aligned}
\pi_{\langle\chi, \tau\rangle}(\hat{x}, \hat{\theta}) & \equiv \sum_{i \in I}\left(\mathbb{E}\left[v_{i}\left(\chi\left(\hat{\theta}_{i}, \widetilde{\theta}_{-i}\right), \hat{\theta}_{i}, \widetilde{\theta}_{-i}\right)+\tau_{i}\left(\hat{\theta}_{i}, \widetilde{\theta}_{-i}\right)\right]-\mathbb{E}\left[v_{i}\left(\hat{x}, \hat{\theta}_{i}, \widetilde{\theta}_{-i}\right)\right]-\mathbb{E}\left[\tau_{i}(\widetilde{\theta})\right]\right) \\
\bar{\pi}_{\langle\chi, \tau\rangle}(\hat{x}) & \equiv \inf _{\hat{\theta} \in \Theta} \pi_{\langle\chi, \tau\rangle}(\hat{x}, \hat{\theta}) .
\end{aligned}
$$

The function $\pi_{\langle\chi, \tau\rangle}(\hat{x}, \hat{\theta})$ can be interpreted as the expected profit of an intermediary who runs the mechanism $\langle\chi, \tau\rangle$, incurring expected cost $\mathbb{E}\left[\tau_{i}(\widetilde{\theta})\right]$ for each agent $i$, and charges each agent $i$ a participation fee $\left(\mathbb{E}\left[v_{i}\left(\chi\left(\hat{\theta}_{i}, \widetilde{\theta}_{-i}\right), \hat{\theta}_{i}\right)+\tau_{i}\left(\hat{\theta}_{i}, \widetilde{\theta}_{-i}\right)\right]-\right.$ $\left.\mathbb{E}\left[v_{i}\left(\hat{x}, \hat{\theta}_{i}, \widetilde{\theta}_{-i}\right)\right]\right)$ that makes his type $\hat{\theta}_{i}$ indifferent between participating in the mechanism and getting the status quo $(\hat{x}, 0)$. The function $\bar{\pi}_{\langle\chi, \tau\rangle}(\hat{x})$ is therefore the expected profit of an intermediary who runs mechanism $\langle\chi, \tau\rangle$ with status quo $(\hat{x}, 0)$ and charges the maximal participation fees that assure participation of all types.

We begin with a lemma that has a number of (more restrictive) precedents for efficient decision rules: 6

Lemma 1. If $\langle\chi, \tau\rangle$ is an IC mechanism and $\hat{x} \in X$ satisfies $\bar{\pi}_{\langle\chi, \tau\rangle}(\hat{x}) \geq 0$, then status quo decision $\hat{x}$ sustains decision rule $\chi$ in bargaining.

Proof. Let $(\chi, \bar{\tau})$ be the IC BB mechanism with transfers (2). For each $i \in I$ and each $\theta \in \Theta$, let

$$
\hat{\tau}_{i}(\theta)=\bar{\tau}_{i}(\theta)-K_{i}+\frac{1}{|I|} \sum_{j \in I} K_{j}
$$

where, for all $i \in I$,

$$
K_{i}=\inf _{\hat{\theta}_{i} \in \Theta_{i}}\left(\mathbb{E}\left[v_{i}\left(\chi\left(\hat{\theta}_{i}, \widetilde{\theta}_{-i}\right), \hat{\theta}_{i}, \widetilde{\theta}_{-i}\right)+\bar{\tau}_{i}\left(\hat{\theta}_{i}, \widetilde{\theta}_{-i}\right)\right]-\mathbb{E}\left[v_{i}\left(\hat{x}, \hat{\theta}_{i}, \widetilde{\theta}_{-i}\right)\right]\right)
$$

Mechanism $\langle\chi, \hat{\tau}\rangle$ inherits IC and BB from mechanism $(\chi, \bar{\tau})$, and satisfies IR for status quo $(\hat{x}, 0)$ by construction of $K_{i}$ and the fact that

$$
\sum_{j \in I} K_{j}=\bar{\pi}_{\langle\chi, \bar{\tau}\rangle}(\hat{x})=\bar{\pi}_{\langle\chi, \tau\rangle}(\hat{x}) \geq 0 .
$$

(The first equality holds since transfers $\bar{\tau}$ satisfy (BB), and the second equality holds since by (2), we have $\mathbb{E}\left[\bar{\tau}_{i}\left(\hat{\theta}_{i}, \widetilde{\theta}_{-i}\right)-\bar{\tau}_{i}(\widetilde{\theta})\right]=\mathbb{E}\left[\tau_{i}\left(\hat{\theta}_{i}, \widetilde{\theta}_{-i}\right)-\tau_{i}(\tilde{\theta})\right]$.)

We now use this lemma along with Proposition 1 to establish a possibility result.

\footnotetext{
${ }^{6}$ Precedents can be found, e.g., in Makowski and Mezzetti (1994), Krishna and Perry (1998), Neeman (1999), Williams (1999), Schweizer (2006), Che (2006), and Figueroa and Skreta (2007). However, these precedents focus on efficient decision rules, and make restrictive assumptions on type spaces and utility functions to show that the condition is necessary as well as sufficient for implementing efficient outcomes. (We state such a necessity result in Lemma 3 below.)
} 
Proposition 2. Suppose that the decision space $X$ is a convex set in a topological vector space and the total surplus $s(x, \theta)$ is convex in the decision $x \in X$ for each state $\theta \in \Theta$. Then if decision rule $\chi$ is implementable in an IC mechanism that satisfies CC, the status quo decision equal to the expected equilibrium decision $\mathbb{E}[\chi(\widetilde{\theta})]$ sustains $\chi$ in bargaining.

Proof. Let $\langle\chi, \tau\rangle$ be an IC and CC mechanism, and let $(\widetilde{x}, \tilde{t})$ be a random variable equal in distribution to $(\chi(\widetilde{\theta}), \tau(\widetilde{\theta}))$ and independent of it. The convexity of $s(x, \theta)$ in $x$ implies that for any $\hat{\theta} \in \Theta, \pi_{\langle\chi, \tau\rangle}(\hat{x}, \hat{\theta})$ is concave in $\hat{x}$, and therefore by Jensen's inequality,

$$
\begin{aligned}
\pi_{\langle\chi, \tau\rangle}(\mathbb{E}[\chi(\tilde{\theta})], \hat{\theta}) & \geq \mathbb{E}\left[\pi_{\langle\chi, \tau\rangle}(\tilde{x}, \hat{\theta})\right] \\
& =\sum_{i \in I}\left(\mathbb{E}\left[v_{i}\left(\chi\left(\hat{\theta}_{i}, \widetilde{\theta}_{-i}\right), \hat{\theta}_{i}, \widetilde{\theta}_{-i}\right)+\tau_{i}\left(\hat{\theta}_{i}, \widetilde{\theta}_{-i}\right)\right]-\mathbb{E}\left[v_{i}\left(\widetilde{x}, \hat{\theta}_{i}, \widetilde{\theta}_{-i}\right)\right]-\mathbb{E}\left[\widetilde{t}_{i}\right]\right),
\end{aligned}
$$

where we used the fact that $\mathbb{E}\left[\tilde{t}_{i}\right]=\mathbb{E}\left[\tau_{i}(\tilde{\theta})\right]$. Each summation term is nonnegative by Proposition 1(a), since it can be viewed as the expected utility of agent $i$ 's type $\hat{\theta}_{i}$ in the mechanism $\langle\chi, \tau\rangle$ net of his expected utility at the randomized status quo $(\tilde{x}, \tilde{t})$. Apply Lemma 1.

\subsection{Application to private values}

In the case of private values, in which utilities take the form $v_{i}\left(x, \theta_{i}\right)$, any efficient decision rule (1) can be implemented in a dominant-strategy incentive-compatible direct mechanism [Vickrey-Clarke-Groves (VCG) mechanism] $\eta^{*}=\left\langle\chi^{*}, \tau^{*}\right\rangle$ with transfers

$$
\tau_{i}^{*}(\theta)=\sum_{j \in I \backslash\{i\}} v_{j}\left(\chi^{*}(\theta), \theta_{j}\right)
$$

Furthermore, in the private-values setting, any decision rule trivially satisfies CC. Hence, Proposition 2 yields the following corollary.

Corollary 1. Suppose that there are private values, the decision space $X$ is a convex set in a topological vector space, and the total surplus $s(x, \theta)$ is convex in the decision $x \in X$ for each state $\theta \in \Theta$. Then if $\chi^{*}$ is an efficient decision rule, the status quo decision equal to the expected efficient decision $\hat{x}=\mathbb{E}\left[\chi^{*}(\widetilde{\theta})\right]$ permits efficient bargaining.

For example, in the symmetric partnership model of Cramton et al. (1987), equal ownership shares is the expected efficient decision and Corollary 1 tells us that this status quo decision permits efficient bargaining, consistent with their results. More generally, any symmetric model has a symmetric expected efficient decision, and under the corollary's convexity assumptions, this status quo permits efficient bargaining, as in Yenmez (2007) and Gershkov and Schweinzer (2010).

\subsection{Application to interdependent values}

The case of interdependent values involves two complications: an efficient decision rule (a) need not be implementable in an IC mechanism (as noted, e.g., by Jehiel and 
Moldovanu 2001) and (b) need not satisfy CC. However, in the special case of onedimensional types, there exist simple "single-crossing" conditions that ensure CC and IC implementability. ${ }^{7}$

Lemma 2. Suppose that $\Theta_{i} \subseteq \mathbb{R}$ for each $i \in I$, then the following results hold.

(a) Any decision rule $\chi$ such that for each $i \in I, v_{i}\left(\chi\left(\theta_{i}^{\prime}, \theta_{-i}\right), \theta_{i}, \theta_{-i}\right)$ has increasing differences in $\left(\theta_{i}^{\prime}, \theta_{i}\right) \in \Theta_{i} \times \Theta_{i}$ for all $\theta_{-i} \in \Theta_{-i}$, is implementable in an IC mechanism.

(b) Any mechanism $\langle\chi, \tau\rangle$ such that for all $i, j \in I$ with $j \neq i, v_{i}\left(\chi\left(\theta_{j}^{\prime}, \theta_{-j}^{\prime}\right), \theta_{j}, \theta_{-j}\right)$ has increasing differences in $\left(\theta_{j}^{\prime}, \theta_{j}\right) \in \Theta_{j} \times \Theta_{j}$ for all $\theta_{-j}^{\prime}, \theta_{-j} \in \Theta_{-j}$, is $C C$.

Proof. For part (a), fix an agent $i \in I$ and $\theta_{-i} \in \Theta_{-i}$. Consider a single-agent environment with decision space $\Theta_{i}$, decision rule $\hat{\chi}_{i}: \Theta_{i} \rightarrow \Theta_{i}$ given by $\hat{\chi}_{i}\left(\theta_{i}\right)=\theta_{i}$, and the agent's utility function $\hat{v}_{i}\left(\theta_{i}^{\prime}, \theta_{i}\right)=v_{i}\left(\chi\left(\theta_{i}^{\prime}, \theta_{-i}\right), \theta_{i}, \theta_{-i}\right)$. The decision rule $\hat{\chi}_{i}$ is increasing and $\hat{v}_{i}\left(\theta_{i}^{\prime}, \theta_{i}\right)$ has increasing differences by assumption. Hence, by Rochet's (1987) Proposition 1 , there exists a transfer rule $\tau_{i}\left(\cdot, \theta_{-i}\right): \Theta_{i} \rightarrow \mathbb{R}$ that satisfies the agent's incentive constraints in this single-agent environment, which can be written as ${ }^{8}$

$$
v_{i}\left(\chi\left(\theta_{i}, \theta_{-i}\right), \theta_{i}, \theta_{-i}\right)+\tau_{i}\left(\theta_{i}, \theta_{-i}\right) \geq v_{i}\left(\chi\left(\theta_{i}^{\prime}, \theta_{-i}\right), \theta_{i}, \theta_{-i}\right)+\tau_{i}\left(\theta_{i}^{\prime}, \theta_{-i}\right)
$$

for all $\theta_{i}, \theta_{i}^{\prime} \in \Theta_{i}$.

Putting these transfer rules together (for all $\theta_{-i} \in \Theta_{-i}$ and all $i \in I$ ) yields a mechanism $\langle\chi, \tau\rangle$ that is dominant-strategy incentive compatible and therefore IC.

For part (b), apply logic similar to that in part (a) to an agent $i \in I$ who observes the type $\theta_{j}$ of another agent $j \in I \backslash\{i\}$ rather than his own type $\theta_{i}$. The argument implies that for any fixed $\theta_{-j}, \theta_{-j}^{\prime} \in \Theta_{-j}$, the decision rule $\chi\left(\cdot, \theta_{-j}^{\prime}\right): \Theta_{j} \rightarrow X$ is implementable with some transfer rule $\hat{\tau}_{i}\left(\cdot, \theta_{-j}, \theta_{-j}^{\prime}\right): \Theta_{j} \rightarrow \mathbb{R}$, i.e.,

$$
\begin{array}{r}
v_{i}\left(\chi\left(\theta_{j}, \theta_{-j}^{\prime}\right), \theta_{j}, \theta_{-j}\right)+\hat{\tau}_{i}\left(\theta_{j}, \theta_{-j}, \theta_{-j}^{\prime}\right) \geq v_{i}\left(\chi\left(\theta_{j}^{\prime}, \theta_{-j}^{\prime}\right), \theta_{j}, \theta_{-j}\right)+\hat{\tau}_{i}\left(\theta_{j}^{\prime}, \theta_{-j}, \theta_{-j}^{\prime}\right) \\
\quad \text { for all } \theta_{j}, \theta_{j}^{\prime} \in \Theta_{j} .
\end{array}
$$

Now, let $\widetilde{\widetilde{\theta}}_{-j}$ be a random variable with the same distribution as $\widetilde{\theta}_{-j}$ but independent of it. Taking a subset $T \subseteq I \backslash\{i\}$ of agents, substitute $\theta=\left(\theta_{i}, \widetilde{\theta}_{-i}\right)$ and $\theta^{\prime}=\left(\theta_{i}^{\prime}, \widetilde{\theta}_{T}, \widetilde{\widetilde{\theta}}_{I \backslash(T \cup\{i\})}\right)$ in the above inequality, and take expectations to obtain (note that the expectations of transfers cancel out since random variables $\widetilde{\widetilde{\theta}}_{j}$ and $\widetilde{\theta}_{j}$ have the same distribution and are independent of the other variables)

$$
\mathbb{E}\left[v_{i}\left(\chi\left(\theta_{i}^{\prime}, \widetilde{\theta}_{T \cup\{j\}}, \widetilde{\widetilde{\theta}}_{I \backslash(T \cup\{i, j\})}\right), \theta_{i}, \widetilde{\theta}_{-i}\right)\right] \geq \mathbb{E}\left[v_{i}\left(\chi\left(\theta_{i}^{\prime}, \widetilde{\theta}_{T}, \widetilde{\widetilde{\theta}}_{I \backslash(T \cup\{i\})}\right), \theta_{i}, \widetilde{\theta}_{-i}\right)\right] .
$$

\footnotetext{
${ }^{7}$ Below we use the concepts of (strictly) increasing differences, supermodularity, and lattices, whose definitions can be found in Topkis (1998).

${ }^{8}$ Rochet's (1987) Proposition 1 assumes that the agent's utility function is twice continuously differentiable and his type space is a closed interval, but it is clear from his proof that these assumptions are not needed. The sufficiency part of his proposition, which we use here, relies only on the increasing difference property of the agent's utility function (the necessity part uses the stronger property of strictly increasing differences).
} 
This means that $\mathbb{E}\left[v_{i}\left(\chi\left(\theta_{i}^{\prime}, \widetilde{\theta}_{T}, \widetilde{\widetilde{\theta}}_{I \backslash(T \cup\{i\})}\right), \theta_{i}, \widetilde{\theta}_{-i}\right)\right]$ is nondecreasing in $T \subseteq I \backslash\{i\}$. Comparing $T=\varnothing$ to $T=I \backslash\{i\}$ yields CC.

While both parts of Lemma 2 have precedents in the literature, we state them in greater generality and highlight a connection between them. Part (a) of the lemma states that increasing differences in agents' utilities between their true and reported types ensure implementability, which extends an observation of García (2005) (discarding unnecessary assumptions such as smoothness of utility functions and connectedness of type spaces). The derivation of part (b) applies the same approach to hypothetical situations in which agent $i$ observes and reports the type $\theta_{j}$ of another agent $j \neq i$ rather than his own type. Increasing differences between the report $\theta_{j}^{\prime}$ and the true type $\theta_{j}$ in agent $i$ 's utility imply that his truthful reporting of $\theta_{j}$ could be sustained by some transfers. In turn, using an observation of Rahman (2010, Theorem 1), this implies that agent $i$ 's expected nonmonetary utility could not be improved by any randomized "undetectable deviation," i.e., deviation to a randomized reporting strategy in which his report $\theta_{j}^{\prime}$ has the same distribution as the true type $\theta_{j}$. This explains the satisfaction of CC in this setting. ${ }^{9}$

Lemma 2 together with Proposition 2 yields the following corollary.

Corollary 2. Suppose that the decision space $X$ is a convex set in a topological vector space and the total surplus $s(x, \theta)$ is convex in the decision $x \in X$ for each state $\theta \in \Theta$, and that $\Theta_{i} \subseteq \mathbb{R}$ for each $i \in I$. Then for any decision rule $\chi$ such that for all $i, j \in I$, $v_{i}\left(\chi\left(\theta^{\prime}\right), \theta\right)$ has increasing differences in $\left(\theta_{j}^{\prime}, \theta_{j}\right) \in \Theta_{j} \times \Theta_{j}$ for all $\theta_{-j}^{\prime}, \theta_{-j} \in \Theta_{-j}$, the status quo decision equal to the expected equilibrium decision $\mathbb{E}[\chi(\widetilde{\theta})]$ sustains $\chi$ in bargaining.

The following example describes a setting with interdependent values in which the desired increasing difference conditions are satisfied by an efficient decision rule, so that Corollary 2 establishes the possibility of efficient bargaining.

ExAmple 1. Let $X=\left\{x \in \mathbb{R}_{+}^{I}: \sum_{i \in I} x_{i}=1\right\}$, where $x_{i}$ is interpreted as agent $i$ 's consumption of a good whose total supply is 1 and, for all $i \in I, \Theta_{i} \subseteq \mathbb{R}$ and $v_{i}(x, \theta)=\phi_{i}(\theta) x_{i}$, where $\phi_{i}: \Theta \rightarrow \mathbb{R}$ is a differentiable function. Consider first the case of $I=2$, and suppose that for each $i=1,2, \partial \phi_{i}(\theta) / \partial \theta_{i}-\partial \phi_{-i}(\theta) / \partial \theta_{i}>0$. Then an efficient decision rule $\chi_{i}^{*}(\theta)$ is nondecreasing in $\theta_{i}$ and nonincreasing in $\theta_{-i}$. If, furthermore, $\partial \phi_{i}(\theta) / \partial \theta_{i} \geq 0 \geq \partial \phi_{-i}(\theta) / \partial \theta_{i}$ for each $i$, decision rule $\chi^{*}$ satisfies the increasing difference conditions of Corollary $2 .^{10}$ Since the surplus $s(x, \theta)$ is linear in the decision $x$ and the decision set $X$ is convex, the status quo decision equal to the expected efficient decision $\mathbb{E}\left[\chi^{*}(\widetilde{\theta})\right]$ permits efficient bargaining.

This conclusion extends to the case of $I>2$ agents whose values take the separable form $\phi_{i}(\theta)=g_{i}\left(\theta_{i}\right)+\sum_{j \in I \backslash\{i\}} h_{j}\left(\theta_{j}\right)$. In this case, efficiency (1) means that $\chi_{i}^{*}(\theta)>0$

\footnotetext{
${ }^{9}$ An alternative explanation of CC follows from a property of supermodular functions noted by Milgrom and Roberts (1995, p. 186).

${ }^{10}$ Namely, $\chi_{i}^{*}(\theta)$ being nondecreasing in $\theta_{i}$ and $\partial \phi_{i}(\theta) / \partial \theta_{i} \geq 0$ imply the condition of Lemma 2(a), which ensures that $\chi^{*}$ is implementable in an IC mechanism, while $\chi_{i}^{*}(\theta)$ being nonincreasing in $\theta_{-i}$ and $\partial \phi_{-i}(\theta) / \partial \theta_{i} \leq 0$ imply the condition of Lemma 2(b), which ensures that the mechanism is CC.
} 
only for $i \in \arg \max _{j \in J}\left[g_{j}\left(\theta_{j}\right)-h_{j}\left(\theta_{j}\right)\right]$. Thus, when $g_{i}^{\prime}>h_{i}^{\prime}$ for all $i \in I$, there again exists an efficient decision rule $\chi^{*}$ in which $\chi_{i}^{*}(\theta)$ is nondecreasing in $\theta_{i}$ and nonincreasing in $\theta_{-i}$. (For the latter monotonicity, tie-breaking should not depend on the types of agents who do not get the object.) If, furthermore, $g_{i}^{\prime} \geq 0 \geq h_{i}^{\prime}$ for all $i \in I$, then the decision rule $\chi^{*}$ satisfies the increasing difference assumptions of Corollary 2 . Since the convexity assumptions of Corollary 2 also hold, the status quo decision equal to the expected efficient decision $\mathbb{E}\left[\chi^{*}(\widetilde{\theta})\right]$ permits efficient bargaining.

The conclusion of the separable part of Example 1 generalizes Theorem 3 of Fieseler et al. (2003), which shows that in the symmetric version of the example, the symmetric status quo $(1 /|I|, \ldots, 1 /|I|)$ permits efficient bargaining. The example can also be used to demonstrate the importance of CC for the existence of an efficiency-permitting status quo. Namely, Fieseler et al. (2003, Theorem 4) show that in the symmetric version of the separable case of Example 1, when $g^{\prime}>h^{\prime}>0$, there exists a distribution over types for which no status quo permits efficient bargaining. In this case, all of the assumptions of Corollary 2 except for CC are satisfied by an efficient decision rule (in particular, it is implementable in an IC mechanism, as noted in footnote 10). ${ }^{11}$

Example 1 can also be used to interpret CC. Observe that the condition can be interpreted as saying that a "naive" agent who knows the distribution of others' messages but believes these messages to be independent of their types underestimates his expected utility in the mechanism (for any type observed and any message sent). (This concept of naivete is examined by Eyster and Rabin 2005, who study its behavioral effects, in contrast to our analysis.) In Example 1, a naive agent $i$ believes his expected value for a received good to be $\mathbb{E}\left[\phi_{i}\left(\theta_{i}, \widetilde{\theta}_{-i}\right)\right]$, failing to condition the expectation on the fact that he receives the good only when other agents observe sufficiently low signals $\widetilde{\theta}_{-i}$. Thus, when $\phi_{i}(\theta)$ is decreasing in $\theta_{-i}$, so CC holds, a naive agent who receives the good is, on average, pleasantly surprised by its value to him. This situation can be referred to as winner's blessing (see Fieseler et al. 2001), in contrast to the more familiar winner's curse case in which $\phi_{i}(\theta)$ is increasing in $\theta_{-i}$ and so a naive agent is, on average, negatively surprised by the received good's value. Thus, condition CC, which postulates excessive pessimism of naive agents, can be viewed as a formalization of winner's blessing for general mechanism design settings.

In contrast to the special setting of Example 1, the satisfaction of CC (and thus the possibility of efficient bargaining) is not generally determined by agents' marginal utilities for the decision being nonincreasing in the others' types. For an illustration, consider the following example, in which marginal utilities for decisions are increasing in others' types, yet an efficient decision rule satisfies CC and efficient bargaining is possible.

ExAmple 2. Suppose that $X$ is a lattice, $\Theta_{i} \subseteq \mathbb{R}$ for all $i \in I$, each agent $i$ 's utility $v_{i}(x, \theta)$ has increasing differences in $(x, \theta)$, and the total surplus $s(x, \theta)$ has strictly increasing differences in $(x, \theta)$ and is supermodular in $x \in X$ in each state $\theta \in \Theta$. (This setting can

\footnotetext{
${ }^{11}$ A related asymmetric example with one-sided private information violating CC in which no status quo permits efficient bargaining is offered by Jehiel and Pauzner (2006) in their Corollary 3(iii).
} 
be interpreted as provision of a public good or a set of complementary public goods, with types raising all agents' incremental utilities for all the goods.) Then by Topkis (1998, Theorem 2.8.4), any efficient decision rule $\chi^{*}$ is nondecreasing, which implies the increasing difference conditions of Corollary 2 . Hence, if the convexity conditions of Corollary 2 hold as well, then the status quo decision equal to the expected efficient decision $\mathbb{E}\left[\chi^{*}(\widetilde{\theta})\right]$ permits efficient bargaining. (For example, all of the required conditions hold when $X$ is a convex sublattice of $\mathbb{R}^{m}$, and for each $i \in I, v_{i}(x, \theta)=\phi_{i}(\theta) \cdot x$, where $\phi_{i}: \Theta \rightarrow \mathbb{R}^{m}$ is a strictly increasing function.)

\section{Remarks}

In this section, we make a few remarks on our results, concentrating on the special case of quasilinear environments with private values studied in Section 3, which has been the focus of most of the literature.

\subsection{Comparison to Schweizer's (2006) Proposition 2}

Our derivation of Proposition 2 can be interpreted as follows. Fix an IC and CC mechanism $\langle\chi, \tau\rangle$ and consider the zero-sum game in which an "intermediary" chooses the status quo decision $\hat{x} \in X$ to maximize $\pi_{\langle\chi, \tau\rangle}(\hat{x}, \hat{\theta})$ in (3) and an "adversary" chooses the "critical types" $\hat{\theta} \in \Theta$ to minimize it. According to Lemma 1, if status quo decision $\hat{x}$ guarantees the intermediary a nonnegative payoff regardless of the adversary's action, then it sustains decision rule $\chi$ in bargaining. Now, by randomizing over status quo decisions with the same distribution as the equilibrium decision rule $\chi(\widetilde{\theta})$, the intermediary guarantees himself a nonnegative expected payoff, since by Proposition 1(a), the intermediary's expected profit on each agent-given by the expectation of the corresponding summation term in (3)-would be nonnegative at any $\hat{\theta} \in \Theta$. Finally, if the intermediary's payoff is concave in $\hat{x}$ [which is true if $s(x, \theta)$ is convex] and his choice set $X$ is convex, the randomization can be replaced with its expectation $\mathbb{E}[\chi(\widetilde{\theta})]$ without reducing the intermediary's expected payoff. Therefore, by Lemma 1 , the status quo $\mathbb{E}[\chi(\widetilde{\theta})]$ sustains decision rule $\chi$ in bargaining.

Schweizer (2006) focuses on the private-value setting and an efficient decision rule (1), which is then implementable in the VCG mechanism $\eta^{*}=\left\langle\chi^{*}, \tau^{*}\right\rangle$ with transfers (4). In this case, letting $S(\theta) \equiv \max _{x \in X} s(x, \theta)$, the intermediary's payoff (3) can be written as

$$
\pi_{\eta^{*}}(\hat{x}, \hat{\theta})=-(|I|-1) \mathbb{E}[S(\widetilde{\theta})]+\sum_{i \in I} \mathbb{E}\left[S\left(\hat{\theta}_{i}, \widetilde{\theta}_{-i}\right)\right]-s(\hat{x}, \hat{\theta}) .
$$

He makes a number of assumptions to ensure that the resulting simultaneous zerosum game between the intermediary and the adversary has a pure-strategy Nash equilibrium-a "saddle point" $\left(\hat{x}^{\circ}, \hat{\theta}^{\circ}\right)$. In this case, strategy $\hat{x}^{\circ}$ is the intermediary's "maximin" strategy and guarantees him at least the saddle-point payoff $\pi_{\eta^{*}}\left(\hat{x}^{\circ}, \hat{\theta}^{\circ}\right)$ regardless of the adversary's action (Rockafellar 1970). Schweizer then shows that the saddle-point payoff is nonnegative, a conclusion that can also be seen from the observation that the intermediary cannot improve upon the saddle-point payoff by any 
mixed strategy, in particular by the randomization $\chi(\widetilde{\theta})$, which would guarantee him a nonnegative payoff by Proposition 1(a). Hence, by Lemma 1, status quo $\hat{x}^{\circ}$ permits efficient bargaining.

To ensure the existence of a saddle point, Schweizer makes stronger assumptions than those of our Proposition 2. In addition to our assumptions, he assumes that the total surplus is linear in $\theta$ [which ensures that the payoff (5) is convex in the adversary's action $\hat{\theta}$, that the adversary's choice set $\Theta$ is convex, and that the sets $X$ and $\Theta$ are compact subsets of Euclidean spaces, and also makes some continuity assumptions. ${ }^{12}$ Our approach dispenses with the extra assumptions-in particular, we allow for infinite-dimensional decisions or types, nonconvex or noncompact type spaces, and utility functions that are nonlinear or discontinuous. The only indispensable assumptions prove to be convexity of the total surplus in the decision and of the decision set $X$ (and even these assumptions are not needed if a randomized status quo is allowed). A second and perhaps more important advantage of our approach is our explicit description of a natural status quo that permits efficient bargaining: the expected efficient decision. ${ }^{13}$

On the other hand, Schweizer's saddle-point status quo or, more generally, the "maximin" status quo, which maximizes the intermediary's expected profit, is of some independent interest. This expected profit-maximizing status quo generally differs from the expected equilibrium decision, as we illustrate in Example 3 below. For analyzing this example, we write the two best-response conditions that characterize a saddle point $\left(\hat{x}^{\circ}, \hat{\theta}^{\circ}\right)$ of the intermediary's payoff (3) in a general mechanism $\langle\chi, \tau\rangle$ for private-value settings in the form

$$
\begin{aligned}
& \hat{\theta}_{i}^{\circ} \underset{\hat{\theta}_{i} \in \Theta_{i}}{\arg \min } \mathbb{E}\left[v_{i}\left(\chi\left(\hat{\theta}_{i}, \widetilde{\theta}_{-i}\right), \hat{\theta}_{i}\right)+\tau_{i}\left(\hat{\theta}_{i}, \widetilde{\theta}_{-i}\right)-v_{i}\left(\hat{x}^{\circ}, \hat{\theta}_{i}\right)\right] \quad \text { for all } i \in I \\
& \hat{x}^{\circ} \in \underset{\hat{x} \in X}{\arg \min } s\left(\hat{x}, \hat{\theta}^{\circ}\right) .
\end{aligned}
$$

Condition (6) says that the adversary chooses each agent $i$ 's critical type $\hat{\theta}_{i}^{\circ}$ to minimize the agent's net expected surplus over the status quo decision $\hat{x}^{\circ}$ in mechanism $\langle\chi, \tau\rangle$, while (7) says that the intermediary chooses status quo decision $\hat{x}^{\circ}$ to minimize the total surplus at the critical types $\hat{\theta}^{\circ}$.

ExAmple 3. Suppose that $I=2$, each $\widetilde{\theta}_{i}$ is distributed on $\Theta_{i}=[0,1]$ according to a strictly increasing cumulative distribution function $F_{i}$, the decision space is $X=$

\footnotetext{
${ }^{12}$ His footnotes 1 and 2 state some continuity and smoothness assumptions, although they could be relaxed for the purpose of proving the existence of a saddle point.

${ }^{13}$ The saddle-point status quo decision $\hat{x}^{\circ}$ does offer one advantage when mechanism $\langle\chi, \tau\rangle$ is dominantstrategy incentive compatible (as is the VCG mechanism $\eta^{*}$ considered by Schweizer): By using transfers $\hat{\tau}_{i}(\theta \mid \hat{x})=\tau_{i}(\theta)-\left(\left[v_{i}\left(\chi\left(\hat{\theta}_{i}^{\circ}, \theta_{-i}\right), \hat{\theta}_{i}^{\circ}\right)-\tau_{i}\left(\hat{\theta}_{i}^{\circ}, \theta_{-i}\right)\right]-v_{i}\left(\hat{x}, \hat{\theta}_{i}^{\circ}\right)\right)$ with $\hat{x}=\hat{x}^{\circ}$, the intermediary guarantees a nonnegative budget surplus not just in expectation, but in every state $\theta$. Indeed, by the saddlepoint condition (7) below and the dominant-strategy incentive compatibility of $\langle\chi, \tau\rangle, \sum_{i \in I} \hat{\tau}_{i}\left(\theta \mid \hat{x}^{\circ}\right) \leq$ $\sum_{i \in I} \hat{\tau}_{i}(\theta \mid \chi(\theta)) \leq 0$ for all $\theta \in \Theta$. Furthermore, unlike the balanced-budget mechanism constructed in the proof of Lemma 1, the mechanism $\langle\chi, \hat{\tau}\rangle$ inherits the dominant-strategy incentive compatibility of $\langle\chi, \tau\rangle$. Note, however, that the mechanism $\langle\chi, \hat{\tau}\rangle$ would generally satisfy all of the participation constraints with status quo decision $\hat{x}^{\circ}$ only in expectation, not in the ex post sense.
} 
$\left\{\left(x_{1}, x_{2}\right) \in \mathbb{R}_{+}^{2}: x_{1}+x_{2}=1\right\}$, and $v_{i}\left(x, \theta_{i}\right)=\theta_{i} x_{i}$. Consider any efficient decision rule (1), which has $\chi_{i}^{*}(\theta)=1$ whenever $\theta_{i}>\theta_{-i}$. In this setting there is a unique saddle point, which is found as follows: Given a status quo decision $\hat{x}^{\circ}=\left(\hat{x}_{1}^{\circ}, \hat{x}_{2}^{\circ}\right)$, condition (6) is satisfied only by the types $\hat{\theta}_{i}^{\circ}$ whose expected consumption equals $\hat{x}_{i}^{\circ}$ (any other type can obtain a strictly greater interim net expected utility than that of type $\hat{\theta}_{i}^{\circ}$ by pretending to be type $\left.\hat{\theta}_{i}^{\circ}\right)$. With the efficient decision rule, this means that $F_{-i}\left(\hat{\theta}_{i}^{\circ}\right)=\hat{x}_{i}^{\circ}$. To satisfy condition (7), we must have $\hat{\theta}_{1}^{\circ}=\hat{\theta}_{2}^{\circ}$ [unless $\hat{x}_{i}^{\circ}=1$ for some $i$ and $\hat{\theta}_{i}^{\circ}<\hat{\theta}_{-i}^{\circ} \leq 1$, but this contradicts the previous condition $F_{-i}\left(\hat{\theta}_{i}^{\circ}\right)=\hat{x}_{i}^{\circ}=1$ ]. The equation $F_{1}\left(\hat{\theta}^{\circ}\right)+F_{2}\left(\hat{\theta}^{\circ}\right)=1$ then uniquely defines the saddle-point types $\hat{\theta}_{1}^{\circ}=\hat{\theta}_{2}^{\circ}=\hat{\theta}^{\circ}$ and the status quo decision $\hat{x}^{\circ}=\left(F_{2}\left(\hat{\theta}^{\circ}\right), F_{1}\left(\hat{\theta}^{\circ}\right)\right)$. [This decision was used, for example, by Schmitz (2002) in proving his Proposition 3.] Note that this status quo decision does not change if we perturb $F_{1}$ and $F_{2}$ in ways that keep $F_{1}\left(\hat{\theta}^{\circ}\right)$ and $F_{2}\left(\hat{\theta}^{\circ}\right)$ fixed, but such perturbations generally alter the expected efficient decision $\mathbb{E}\left[\chi^{*}(\widetilde{\theta})\right]$.

\subsection{Deterministic status quo without convexity}

When the assumptions of Proposition 2-convexity of the total surplus $s(x, \theta)$ in the decision $x$ and of the decision set $X$-do not hold, there may not exist a deterministic status quo decision that sustains an IC implementable decision rule $\chi$ in bargaining. To understand these settings, we first note that for certain classical settings, a converse to Lemma 1 also holds.

Lemma 3. Suppose that, in a private-value setting, for each agent $i, \Theta_{i}$ is a smoothly connected subset of a Euclidean space and $v_{i}\left(x, \theta_{i}\right)$ is differentiable in $\theta_{i}$, with the gradient $\nabla_{\theta_{i}} v_{i}\left(x, \theta_{i}\right)$ bounded on $X \times \Theta$. Suppose that mechanism $\langle\chi, \tau\rangle$ is IC. Then status quo decision $\hat{x} \in X$ sustains decision rule $\chi$ in bargaining if and only if $\bar{\pi}_{\langle\chi, \tau\rangle}(\hat{x}) \geq 0$.

Proof. The "if" statement is by Lemma 1. For the "only if" statement, note that by the Revenue Equivalence Theorem (see, e.g., Milgrom and Segal 2002, Section 3.1), the expected budget surplus in any IC mechanism that implements decision rule $\chi$ in which the IR of each agent $i$ 's type $\hat{\theta}_{i}$ holds is bounded above by (3). Thus, the expected budget surplus in any IC IR mechanism that implements $\chi$ is bounded above by $\inf _{\hat{\theta} \in \Theta} \pi_{\langle\chi, \tau\rangle}(\hat{x}, \hat{\theta})=\bar{\pi}_{\langle\chi, \tau\rangle}(\hat{x})$. If this value is negative, the mechanism cannot satisfy (BB).

We now use Lemma 3 to examine the possibility of efficient bargaining in some examples with nonconvexities. For a famous example where there is no deterministic status quo decision permitting efficient bargaining, take Example 3 with the decision space restricted to the nonconvex set $X=\{(1,0),(0,1)\}$. Impossibility of efficient bargaining for either status quo decision is established by the Myerson-Satterthwaite Theorem (Myerson and Satterthwaite 1983). Formally, for status quo decision $\hat{x}=\left(\hat{x}_{i}, \hat{x}_{-i}\right)=$ $(1,0)$, the critical types (6) are $\hat{\theta}_{i}^{\circ}(\hat{x})=1$ and $\hat{\theta}_{-i}^{\circ}(\hat{x})=0$, so in calculating (5), we have 
$S\left(\hat{\theta}_{i}^{\circ}(\hat{x}), \theta_{-i}\right)=1=s(\hat{x}, \hat{\theta})$ and $S\left(\hat{\theta}_{-i}^{\circ}(\hat{x}), \theta_{i}\right)=\theta_{i}$. This yields $\bar{\pi}_{\eta^{*}}(\hat{x})=\pi_{\eta^{*}}\left(\hat{x}, \hat{\theta}^{\circ}(\hat{x})\right)=$ $-\mathbb{E}\left[S(\widetilde{\theta})-\widetilde{\theta}_{i}\right]<0 .{ }^{14}$

We now consider some settings with a convex decision space $X$, but where the total surplus $s(x, \theta)$ is not convex in the decision $x$. (Most classical settings assume concave payoffs, reflecting diminishing marginal returns.) Neeman (1999, p. 685), for instance, offers an example with concave payoffs, in which there is no deterministic status quo decision that permits efficient bargaining. Yet, in some concave settings, the expected efficient status quo decision still permits efficient bargaining.

ExAmple 4. Suppose that $X=\mathbb{R}^{K}$ and for each $i, \Theta_{i} \subseteq \mathbb{R}^{K}, \mathbb{E}\left[\tilde{\theta}_{i}\right]=0$, and $v_{i}\left(x, \theta_{i}\right)=$ $\theta_{i} \cdot x-(1 /|I|)\|x\|^{2}{ }^{15}$ Then the efficient decision rule is $\chi^{*}(\theta)=\sum_{i \in I} \theta_{i}$, and $S(\theta)=$ $\frac{1}{2}\left\|\sum_{i \in I} \theta_{i}\right\|^{2}$. Given a status quo decision $\hat{x},(6)$ is solved by type $\hat{\theta}_{i}^{\circ}(\hat{x})=\hat{x}$ for each $i$, and using the statistical independence of agents' types, we can calculate

$$
\bar{\pi}_{\eta^{*}}(\hat{x})=\pi_{\eta^{*}}\left(\hat{x}, \hat{\theta}^{\circ}(\hat{x})\right)=-\frac{1}{2}(|I|-1)\|\hat{x}\|^{2} .
$$

This function is strictly concave in $\hat{x}$ and is maximized at $\hat{x}=0$, the expected efficient decision, which yields value zero. Thus, by Lemma 3 , the expected efficient decision $\mathbb{E}\left[\chi^{*}(\tilde{\theta})\right]=0$ is the unique status quo decision that permits efficient bargaining.

However, as the discussion at the end of the previous subsection indicates, an expected efficient decision generally does not maximize the intermediary's expected profit. As a result, with concave payoffs, efficiency may be possible with some status quo decision, but not with an expected efficient decision.

ExAmple 5. Let $I=2$ and let $\Theta_{i}=X=[0,1]$ for $i=1,2$. Suppose also that $v_{1}\left(x, \theta_{1}\right)=$ $-\theta_{1}^{1-a} x^{a} / a$ and $v_{2}\left(x, \theta_{2}\right)=\theta_{2}^{1-1 / a} x$, with $a>1$. (Agent 1 is the "seller," agent 2 is the "buyer," and $x$ is the buyer's purchase.) Then the efficient decision rule is $\chi^{*}(\theta)=\theta_{1} \theta_{2}^{1 / a}$ and $S(\theta)=((a-1) / a) \theta_{1} \theta_{2}$. Given a status quo decision $\hat{x}$, (6) is solved by types $\hat{\theta}_{1}^{\circ}(\hat{x})=$ $\mathbb{E}\left[\widetilde{\theta}_{2}\right]^{-1 / a} \hat{x}$ and $\hat{\theta}_{2}^{\circ}(\hat{x})=\mathbb{E}\left[\widetilde{\theta}_{1}\right]^{-a} \hat{x}^{a}$, and so

$$
\bar{\pi}_{\eta^{*}}(\hat{x})=\pi_{\eta^{*}}\left(\hat{x}, \hat{\theta}^{\circ}(\hat{x})\right)=-\frac{1}{a} \mathbb{E}\left[\widetilde{\theta}_{1}\right]^{1-a} \hat{x}^{a}+\mathbb{E}\left[\tilde{\theta}_{2}\right]^{1-1 / a} \hat{x}-\left(\frac{a-1}{a}\right) \mathbb{E}\left[\widetilde{\theta}_{2}\right] \mathbb{E}\left[\tilde{\theta}_{1}\right] .
$$

This function is strictly concave in $\hat{x}$ and is maximized at $\hat{x}^{\circ}=\mathbb{E}\left[\tilde{\theta}_{1}\right] \mathbb{E}\left[\tilde{\theta}_{2}\right]^{1 / a}$, which yields value zero. Thus, by Lemma $3, \hat{x}^{\circ}$ is the unique status quo decision that permits efficient bargaining. In particular, efficient bargaining is not permitted by the expected efficient status quo decision $\mathbb{E}\left[\chi^{*}(\widetilde{\theta})\right]=\mathbb{E}\left[\widetilde{\theta}_{1}\right] \mathbb{E}\left[\left(\widetilde{\theta}_{2}\right)^{1 / a}\right]<\hat{x}^{\circ}$ (by Jensen's inequality).

\footnotetext{
${ }^{14}$ For other examples with a nonconvex decision space $X$ in which there is no deterministic status quo that permits efficient bargaining, see Ornelas and Turner (2007) and Turner (2008).

${ }^{15}$ Where $\|x\|^{2}=x \cdot x$. The conclusion of this example extends to any setting in which the agents' utilities are linear-quadratic in $(\theta, x)$ and the total surplus $s(x, \theta)$ is strictly concave in $x$. Indeed, any such setting can be transformed into the model in the example using affine transformations of $x$ and $\theta$, and adding baseline decision-dependent transfers.
} 


\subsection{Relation to holdup models}

Suppose agents first choose unobservable ex ante investments, with each agent $i$ 's private investment choice $a_{i} \in A_{i}$ determining the distribution of his type $\widetilde{\theta}_{i}$. (Any investment costs can be subsumed into utilities.) Let $a^{*}=\left(a_{1}^{*}, \ldots, a_{I}^{*}\right)$ be an efficient investment profile, i.e., one that maximizes the expected total surplus $\mathbb{E}[S(\tilde{\theta}) \mid a]$. Rogerson (1992, Proposition 2) shows that if, following the realizations of agents' types, the agents participate in an "expected externality" mechanism in which all expectations are taken conditional on the efficient investment profile $a^{*}$, this profile is sustained as a Nash equilibrium of the ex ante investment game. Using our Corollary 1 , the mechanism can also satisfy the interim participation constraints (if quitting is possible after investments are taken and agents observe their own types) when the status quo equals the expected efficient decision $\mathbb{E}\left[\chi^{*}(\widetilde{\theta}) \mid a^{*}\right]$. (More generally, any status quo that satisfies the condition in Lemma 1 can be used.) Note that each agent $i$ 's IR and IC constraints will be satisfied for any realization of his type $\theta_{i}$, provided that the other agents have made efficient investments $a_{-i}^{*}$. Thus, the strategies in which each agent $i$ invests $a_{i}^{*}$, and (regardless of his investment) participates in the mechanism and reports truthfully for each realized $\theta_{i}$, form a perfect Bayesian equilibrium of the mechanism. In an optimistic scenario, we could think of the agents specifying only the expected efficient decision as the status quo in the ex ante contract-if they then expect to bargain according to the mechanism, they will choose efficient investments and achieve an efficient allocation.

The use of an expected efficient decision as the status quo is reminiscent of the result of Edlin and Reichelstein (1996). They show that in a two-agent holdup model in which agents engage in full-information Nash bargaining over trade upon observing nonverifiable investments and values, such a status quo sustains efficient investments in equilibrium, provided that payoffs satisfy a separability condition. Our result, however, is formally quite distinct: e.g., the investments here are unobservable, our bargaining mechanism is quite different from Nash bargaining, and the assumptions of convexity and separability are not nested.

\section{REFERENCES}

Arrow, Kenneth J. (1979), "The property rights doctrine and demand revelation under incomplete information." In Economics and Human Welfare (Michael J. Boskin, ed.), 23-39, Academic Press, New York. [113]

Che, Yeon-Koo (2006), "Beyond the Coasian irrelevance: Asymmetric information.” Unpublished notes, Columbia University. [110, 114]

Cramton, Peter, Robert Gibbons, and Paul Klemperer (1987), "Dissolving a partnership efficiently." Econometrica, 55, 615-632. [110, 115]

d'Aspremont, Claude and Louis-André Gérard-Varet (1979), "Incentives and incomplete information." Journal of Public Economics, 11, 25-45. [113]

Edlin, Aaron S. and Stefan Reichelstein (1996), "Holdups, standard breach remedies, and optimal investments.” American Economic Review, 86, 478-501. [123] 
Eyster, Erik and Matthew Rabin (2005), “Cursed equilibrium.” Econometrica, 73, 1623-1672. [118]

Fieseler, Karsten, Thomas Kittsteiner, and Benny Moldovanu (2001), "Partnerships, lemons, and efficient trade.” Working paper, University of Mannheim. [118]

Fieseler, Karsten, Thomas Kittsteiner, and Benny Moldovanu (2003), "Partnerships, lemons, and efficient trade." Journal of Economic Theory, 113, 223-234. [110, 118]

Figueroa, Nicolás and Vasiliki Skreta (2007), "What to put on the table." Documentos de Trabajo, Serie Economía 237, Centro de Economía Aplicada, Departamento de Ingeniería Industrial, Universidad de Chile. [110, 114]

García, Diego (2005), “Monotonicity in direct revelation mechanisms.” Economics Letters, 88, 21-26. [117]

Gershkov, Alex and Paul Schweinzer (2010), "When queueing is better than push and shove." International Journal of Game Theory, 39, 409-430. [110, 115]

Jehiel, Philippe and Benny Moldovanu (2001), "Efficient design with interdependent valuations." Econometrica, 69, 1237-1259. [115, 116]

Jehiel, Philippe and Ady Pauzner (2006), "Partnership dissolution with interdependent values.” RAND Journal of Economics, 37, 1-22. [110, 118]

Krishna, Vijay and Motty Perry (1998), "Efficient mechanism design.” Unpublished paper. [114]

Laffont, Jean-Jacques and E. Maskin (1979), "A differential approach to expected utility maximizing mechanisms." In Aggregation and Revelation of Preferences (Jean-Jacques Laffont, ed.), 289-308, North-Holland, Amsterdam. [109]

Mailath, George J. and Andrew Postlewaite (1990), "Asymmetric information bargaining problems with many agents." Review of Economic Studies, 57, 351-367. [109]

Makowski, Louis and Claudio Mezzetti (1994), "Bayesian and weakly robust first best mechanisms: Characterizations.” Journal of Economic Theory, 64, 500-519. [114]

Milgrom, Paul R. and D. John Roberts (1995), "Complementarities and fit: Strategy, structure, and organizational change in manufacturing." Journal of Accounting and Economics, 19, 179-208. [117]

Milgrom, Paul R. and Ilya Segal (2002), "Envelope theorems for arbitrary choice sets." Econometrica, 70, 583-601. [121]

Myerson, Roger B. and Mark A. Satterthwaite (1983), "Efficient mechanisms for bilateral trading." Journal of Economic Theory, 29, 265-281. [109, 121]

Neeman, Zvika (1999), "Property rights and efficiency of voluntary bargaining under asymmetric information." Review of Economic Studies, 66, 679-691. [110, 114, 122] 
Ornelas, Emanuel and John L. Turner (2007), "Efficient dissolution of partnerships and the structure of control." Games and Economic Behavior, 60, 187-199. [110, 122]

Rahman, David (2010), “Detecting profitable deviations.” Unpublished paper, Department of Economics, University of Minnesota. [117]

Rochet, Jean-Charles (1987), "A necessary and sufficient condition for rationalizability in a quasilinear context." Journal of Mathematical Economics, 16, 191-200. [116]

Rockafellar, R. Tyrrell (1970), Convex Analysis. Princeton University Press, Princeton, New Jersey. [119]

Rogerson, William P. (1992), "Contractual solutions to the hold-up problem." Review of Economic Studies, 59, 777-793. [123]

Schmitz, Patrick W. (2002), "Simple contracts, renegotiation under asymmetric information, and the hold-up problem." European Economic Review, 46, 169-188. [110, 121]

Schweizer, Urs (2006), "Universal possibility and impossibility results." Games and Economic Behavior, 57, 73-85. [110, 114, 119]

Segal, Ilya (1999), “Contracting with externalities.” Quarterly Journal of Economics, 114, 337-388. [111]

Topkis, Donald M. (1998), Supermodularity and Complementarity. Princeton University Press, Princeton, New Jersey. [116, 119]

Turner, John L. (2008), “Dissolving (in)effective partnerships.” Unpublished paper, University of Georgia. [110, 122]

Williams, Steven R. (1999), "A characterization of efficient, Bayesian incentive compatible mechanisms.” Economic Theory, 14, 155-180. [114]

Yenmez, M. Bumin (2007), “Dissolving multi-partnerships efficiently.” Unpublished paper, Carnegie Mellon University. [110, 115]

Submitted 2009-7-1. Final version accepted 2010-7-20. Available online 2010-7-21. 